\title{
Uncertainty and Sensitivity Analysis of Void Reactivity Feedback for 3D BWR Assembly Model
}

\author{
Andrius Slavickas, Raimondas Pabarčius, Aurimas Tonkūnas, and Eugenijus Ušpuras
}

Lithuanian Energy Institute, Breslaujos Str. 3, LT-44403 Kaunas, Lithuania

Correspondence should be addressed to Andrius Slavickas; andrius.slavickas@lei.lt

Received 5 June 2017; Accepted 1 October 2017; Published 25 October 2017

Academic Editor: Manmohan Pandey

Copyright (C) 2017 Andrius Slavickas et al. This is an open access article distributed under the Creative Commons Attribution License, which permits unrestricted use, distribution, and reproduction in any medium, provided the original work is properly cited.

\begin{abstract}
Uncertainty and sensitivity analysis of void reactivity feedback for 3D BWR fuel assembly model is presented in this paper. Uncertainties in basic input data, such as the selection of different cross section library, manufacturing uncertainties in material compositions, and geometrical dimensions, as well as operating data are considered. An extensive modelling of different input data realizations associated with their uncertainties was performed during sensitivity analysis. The propagation of uncertainties was analyzed using the statistical approach. The results revealed that important information on the code predictions can be obtained by analyzing and comparing the codes estimations and their associated uncertainties.
\end{abstract}

\section{Introduction}

BWR fuel assemblies undergo a significant change in axial moderator density from the bottom to the top of assembly as a result of water coolant boiling. Thus a void fraction changes significantly as a function of axial position in the reactor core. In addition, a void fraction profile may change during a fuel assembly lifetime in reactor core due to other factors, such as fuel burnup, control blade position, type of neighbouring fuel assemblies, and assembly position within the core. It is worth mentioning that reactor core physics has become even more complicated in recent years, because modern fuel assemblies have new sophisticated features, such as newly designed inner bypass regions and part length rods, introducing larger uncertainties in relation to axial void distributions and their impact on integral reactor physical parameters [1-3].

The neutronic characteristics, such as the void reactivity coefficient, are traditionally estimated using deterministic lattice transport codes in a 2D geometry. Void reactivity coefficient is defined by a change of the system reactivity caused by the void fraction change in moderator. However, numerical models compiled using deterministic lattice transport codes are not able to take account of a void fraction change in axial direction or any other effect of axial heterogeneity, which exists in BWR fuel assemblies. The void reactivity coefficient is usually estimated by numerical models using averaged void fraction values across the fuel assembly $(0 \%, 40 \%, 80 \%$, or $100 \%)$. Thus, the question is whether the void reactivity coefficient can be estimated precisely by employing a $2 \mathrm{D}$ model and using the average coolant density since effects of fuel assembly axial heterogeneity are excluded from such simulations.

The analysis presented in this paper is the continuation of the work on study of void reactivity coefficient for 3D BWR fuel assembly model [4]. In previous study the void reactivity coefficient was evaluated considering two states: the uneven distribution of two-phase flow, where average void fraction was $40 \%$ (standard average void fraction in a BWR [5]), and even distribution across the whole fuel assembly height with void fraction equal to $100 \%$ (only vapour flow exists). The difference of reactivity between these two states reflects reactivity change during the transition from average operating conditions to loss of coolant accident conditions. However, other considerable changes in fuel assembly, not just void formation, occur during such transition and could have a significant influence on reactivity change. The question is whether the void reactivity feedback can be estimated precisely since only the change of void fraction is considered in the evaluation of such transition. 
The boundary conditions with two density profiles were assumed for void reactivity feedback estimation in this study. These density profiles were taken from Oak Ridge National Laboratory (ORLN) and the US Nuclear Regulatory Commission (NRC) that initiated a multiyear project to investigate the application of burnup credit for BWR fuel [6]. The void reactivity feedback estimated using these density profiles reflects reactivity change during the transition from the average to maximal achieved operating conditions, considering BWR data collected in this project. A more realistic approach is achieved by implementing these moderator density profiles for the evaluation of void reactivity since the change of moderator density occurs in margins of operating conditions and changes of other parameters can be assumed negligible in such case.

There has been a demand from nuclear industry for bestestimate predictions to be provided with their confidence boundary because of safety and regulation reasons. Understanding of uncertainties and their sources is essential when introducing appropriate design margins. For this reason the uncertainty and sensitivity analysis with regard to the estimation of void reactivity feedback is accomplished in this study as well. The uncertainties in basic input data, such as neutron cross section data, manufacturing uncertainties in material compositions, and geometrical dimensions, are considered. The uncertainties in input variables have been taken into account and an extensive modelling of different input data realizations was performed during sensitivity analysis. The propagation of uncertainties was analyzed by employing a statistical method.

\section{Computational Methodology}

2.1. Fuel Assembly Model. The neutron transport calculations were performed using SCALE 6.1 code package [7]. SCALE provides a framework with 89 computational modules, including three deterministic and three Monte Carlo radiation transport solvers that are selected based on the desired solution strategy. In current study, KENO-VI module (Monte Carlo criticality transport module) was used for simulations in 3D geometry.

A modern $10 \times 10$ BWR design, corresponding to an ATRIUM-10 type fuel assembly, with large internal water channel and fuel rods loaded with MOX fuel, was chosen for this study. The numerical model of assembly was compiled by using data of a Nuclear Energy Agency BWR-MOX benchmark $[7,8]$. The benchmark presents the necessary data of geometry and material composition for compilation of $2 \mathrm{D}$ model. 3D model was developed using benchmark data by adding some axial features which exist in BWR fuel assembly design to capture the effects of axial heterogeneities.

Figure 1 shows the layout of the problem and the fuel composition. Fuel assembly consists of 91 fuel rods with 6 different ( $\mathrm{U}-\mathrm{Pu}) \mathrm{UO}_{2}$ mixtures and one $\mathrm{UO}_{2}-\mathrm{Gd}_{2} \mathrm{O}_{3}$ (U-Gd) mixture. $\mathrm{UO}_{2}$ matrix enrichment by $\mathrm{U}-235$ in the MOX rods was assumed to be $0.20 \%$. The plutonium isotopic vector is given in Table 1. The mean fissile $\mathrm{Pu}$ concentration was 3.93 w/o, averaged over both MOX and U-Gd rods.
The U-Gd rods have a density of $9.867 \mathrm{~g} / \mathrm{cm}^{3}$; meanwhile MOX fuel rods have a density of $9.921 \mathrm{~g} / \mathrm{cm}^{3}$. The fuel temperature was set at $627^{\circ} \mathrm{C}$. The density of the Zircaloy cladding and water channel was $6.55 \mathrm{~g} / \mathrm{cm}^{3}$. The temperature of structure material was set to $327^{\circ} \mathrm{C}$.

The radial $\mathrm{Pu}$ enrichment distribution within the assembly is inversely proportional to the moderation efficiency within the assembly. Higher moderation efficiency exists in peripheral fuel rods since the thermal neutron transport from the water between fuel assemblies exists in these rods. Even higher moderation efficiency exists in corner rods since thermal neutron transport from the water comes through two sides of the lattice cell. Thus, there are lower $\mathrm{Pu}$ concentrations in these rods in order to reduce radial power peaking. Similarly, there are lower $\mathrm{Pu}$ concentrations around the water channel, though larger than those at the periphery of the assembly. The U-Gd rods are placed around the water channel to take advantage of the fact that extra moderation in the water channel increases the effectiveness of Gd.

The heterogeneity of BWR assemblies applies axially along the length of an assembly as well. It is usual to have axial blankets of natural uranium in the top and bottom $(15 \mathrm{~cm}$ of fuel rods) to reduce the axial neutron leakage. There may be some part length fuel rods, extending only over the bottom part of the assembly. Grey colour in Figure 1 indicates such fuel rods. Typical axial dimensions for BWR fuel assemblies are about $370 \mathrm{~cm}$ for the total active fuel length and about $200 \mathrm{~cm}$ for the part length fuel rods. The use of part length rods reduces the amount of fuel in the top of assembly, where neutron moderation is less effective and the build-up of $\mathrm{Pu}$ 239 occurs faster. Thus the use of such rods restricts a buildup of fissile material and a reduction of operative margin. The above-mentioned features details of axial geometry were considered in this study. In addition, there are axial variations of Gd and fissile material enrichments in fuel rods, but they were not introduced to numerical models because of a lack of data.

The standard boundary conditions have been reconsidered in 3D model in comparison to 2D model. Mirror reflective boundary conditions are considered for radial directions. Nevertheless the depiction of axial boundary conditions is more complex. The fuel assembly model with additional regions is usually used in order to represent fuel assembly with reflector zones. Thus the region in the size of one fuel assembly width was added in the top and bottom of fuel assembly to depict mirror reflective axial boundaries. The homogenized mixture of structural materials and moderator was used to approximate the structure of materials in these regions. It was assumed that the bottom region water has density corresponding to saturated conditions. Meanwhile the outflow moderator density was assumed for the top region water. 25,000 generations and 25,000 histories per generation were considered for criticality estimation in order to reduce the impact of calculation results uncertainty for the analysis.

2.2. Void Reactivity Feedback. As it has been mentioned, the geometry of BWR assemblies is very heterogeneous. All design features combined with the axial distribution of steam 


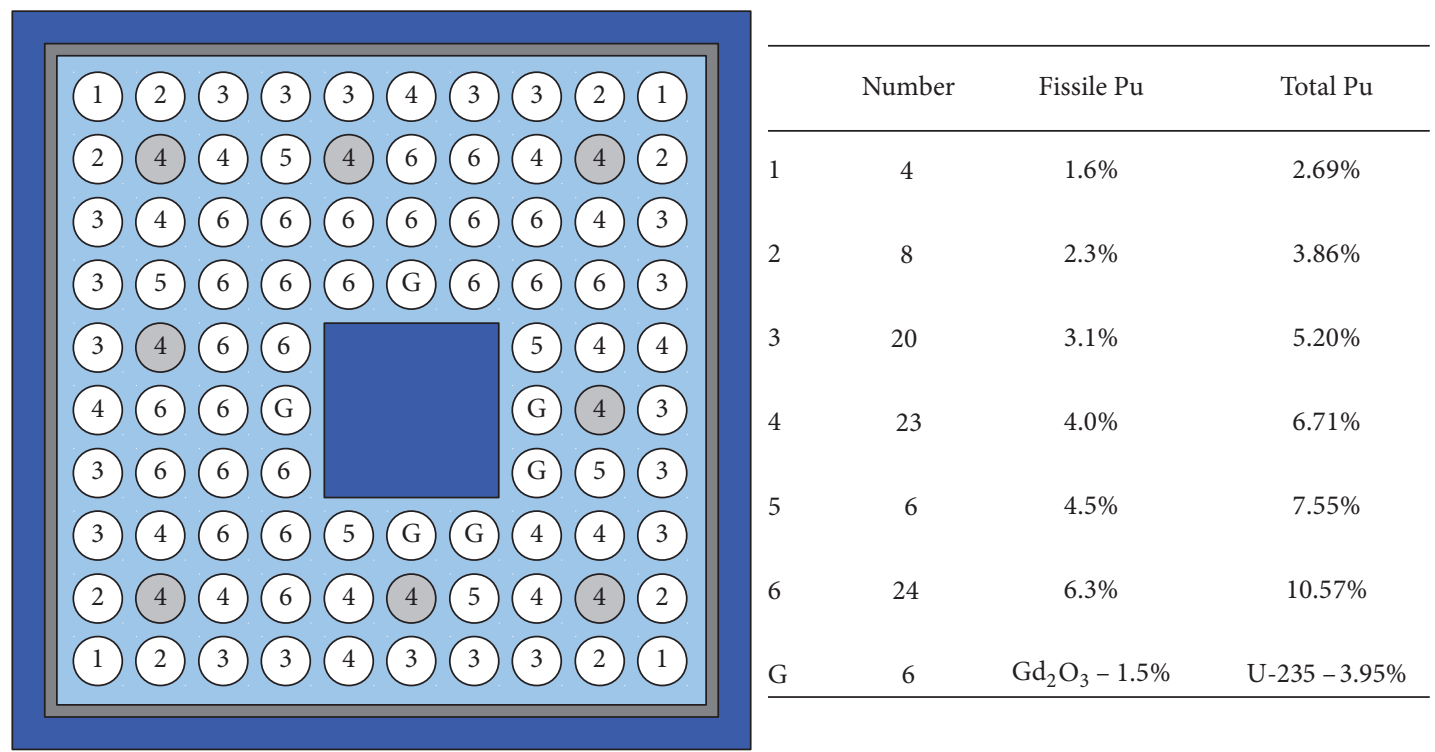

Figure 1: A modern $10 \times 10$ BWR fuel assembly design.

TABLE 1: Plutonium isotopic vector.

\begin{tabular}{lccccc}
\hline $\mathrm{Pu}-238$ & $\mathrm{Pu}-239$ & $\mathrm{Pu}-240$ & $\mathrm{Pu}-241$ & $\mathrm{Pu}-242$ & Fissile $\mathrm{Pu}$ \\
\hline $2.2 \%$ & $46.2 \%$ & $29.4 \%$ & $13.4 \%$ & $8.8 \%$ & $59.6 \%$ \\
\hline
\end{tabular}

voidage (from $0 \%$ at the bottom to $80 \%$ at the top) create a very complex $3 \mathrm{D}$ calculational problem. In addition, there are the bypass regions in BWR assemblies, which are separated from the two-phase flow: central water channel and gaps between the surrounding assemblies. A single-phase flow exists in the bypass regions.

The axial moderator density profile is changing during operating cycles because the assembly power varies due to the depletion of fuel, control blade usage, and change of other core operating parameters. The void reactivity feedback in this study was estimated using two moderator density profiles provided by [6].

The first (minimum density) profile was constructed by selecting the minimum moderator density values in each separate node from all profiles collected in benchmark database. Such approach was considered because this profile represents conservative bounds during normal operation. The second (average density) profile was created by averaging the moderator density values in each separate node across all profiles. The mentioned minimal and average densities profiles are provided in Figure 2. The fluctuation of moderator density in the upper part of fuel assembly is induced by the depressions of coolant for certain axial levels due to the effect of the spacer grids in the fuel assemblies. Single-phase flow density value of $0.75 \mathrm{~g} / \mathrm{cm}^{3}$ was considered for the bypass regions and for inlet coolant.

Void effect is determined by the moderator/fuel ratio, which itself affects two competing neutronic phenomena: the neutron thermalization and thermal neutrons absorption in the moderator. As the moderator/fuel ratio increases the fraction of neutrons reaching thermal energies increases as well.
However, the increase of moderator/fuel ratio also causes larger absorption of thermal neutrons in the moderator. These two phenomena have opposite effects on the fuel lattice infinite multiplication factor (Figure 3). It is seen that at low moderator/fuel ratio region there is an increase of multiplication factor. The phenomena of neutron thermalization dominate over the phenomena of thermal neutrons absorption in the moderator when moderator/fuel ratio increase till multiplication factor reaches the maximum value. This interval of moderator/fuel ratio is the undermoderated region. The void effect in this region is negative since multiplication factor decreases with the decrease of moderator amount (the increase of void). Interval (0.4-1) of moderator/fuel ratio is the overmoderated region because absorption dominates over thermalization. The multiplication value decreases with the increase of the ratio (the decrease of void) and thus positive void effect occurs in overmoderated fuel assembly.

2.3. Estimation of Uncertainties. SUSA software package [9] was used for sensitivity and uncertainty analysis. The software uses approach which is based on a methodology with a statistical basis for assessing uncertainties in neutrons transport code predictions. The uncertainties considered are those resulting from uncertainties in input data variable, such as neutron cross section data, manufacturing uncertainties in material compositions, and geometrical dimensions. A series of KENO-VI calculations, each one of them based on a sample of uncertain input variables generated by SUSA code, produce a set of code predictions variables that can be later statistically treated by SUSA to estimate their uncertainty. 


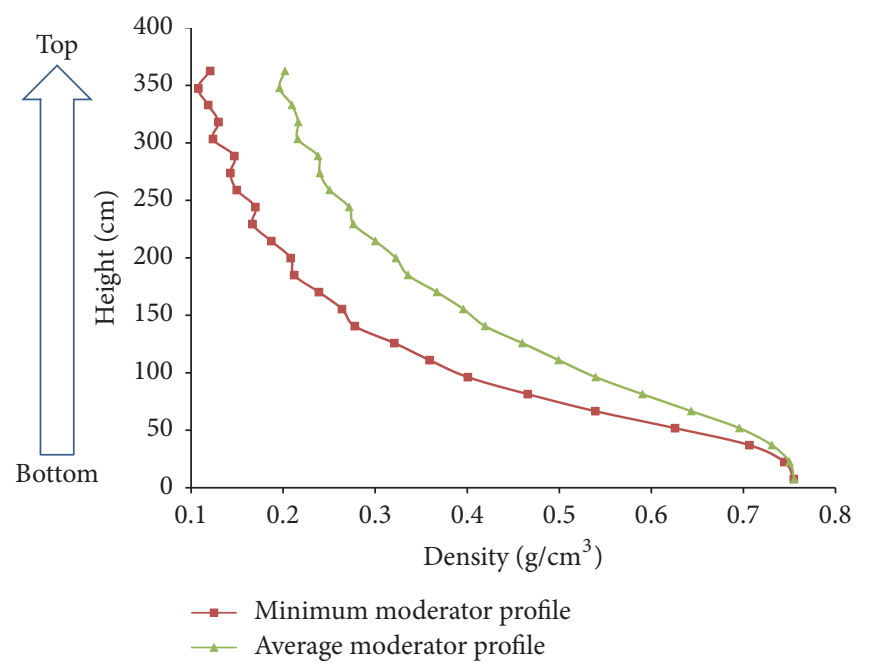

FIgURE 2: Moderator density profiles.

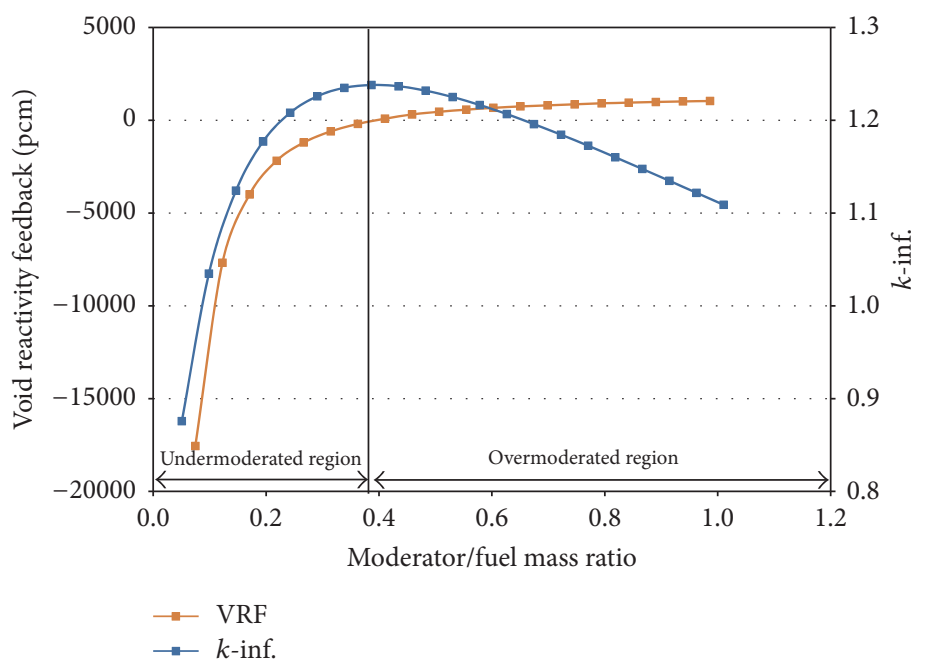

FIGURE 3: Multiplication factor and VRF over the moderator/fuel ratio.

Tolerance intervals measure the uncertainty in the code's output values by providing maximum and minimum values, which contain a certain fraction of possible output values that might result from the uncertainties in the input variables, and give a confidence level for this statement. The size of the sample necessary for a given probability content and confidence level of tolerance intervals, defined by the maximum and minimum values of the output sample, was obtained from nonparametric statistical analysis following the formula of Wilks [10]. For two-sided tolerance limits of $95 \%$ and for $95 \%$ confidence level the minimum sample size $N$ is 93 . Thus 100 simulations of void reactivity feedback were performed in this study to correspond to the desired tolerance limits and confidence level.

The uncertainties of considered variable input data are given in Table 2. SUSA generates probability density functions for each variable. Then statistical sampling techniques, for example, simple random sampling, were employed to generate a series of code calculations with combinations of variable input values randomly selected from their probability density functions. The sample of code output values from executed combinations was later analyzed through software to obtain statistical importance and local and nonparametric sensitivity measures, such as the Spearman's rank correlation coefficients (SRCCs).

One model (parameter number 1) and 8 physical input parameters were considered for the analysis. 44 groups ENDF/B-V and 238 groups ENDV/B-V, ENDV/B-VI, and ENDV/B-VII cross section data libraries were selected for the analysis. The discrete distribution with $25 \%$ probability for each library was chosen. Probability density functions of physical parameters were constructed applying NEA guide [11], which provides supplemental information on manufacturing data of fuel assembly design and reactor operating data. It includes typical uncertainties associated with some parameters as well. In addition, it was assumed that the 
TABLE 2: Uncertainties of input data.

\begin{tabular}{|c|c|c|c|c|c|c|}
\hline $\begin{array}{l}\text { Index of } \\
\text { parameter }\end{array}$ & Variable & Units & Distribution & Min. & Max. & Nominal \\
\hline \multirow{4}{*}{1} & \multirow{4}{*}{$\begin{array}{l}\text { Selection of cross } \\
\text { section library }\end{array}$} & \multicolumn{4}{|c|}{44 ENDF/B-V } & \\
\hline & & \multicolumn{3}{|c|}{238 ENDF/B-V } & & 238 \\
\hline & & \multicolumn{3}{|c|}{238 ENDF/B-VI } & & ENDF/B-VII \\
\hline & & \multicolumn{4}{|c|}{238 ENDF/B-VII } & \\
\hline 2 & Fuel pellet half pitch & $\mathrm{cm}$ & Normal & 0.4335 & 0.4355 & 0.4345 \\
\hline 3 & Cladding half pitch & $\mathrm{cm}$ & Normal & 0.5000 & 0.5050 & 0.5025 \\
\hline 4 & Fuel pellet density & & Normal & $99 \% \times X$ & $101 \% \times \mathrm{X}$ & $\mathrm{X}$ \\
\hline 5 & Enrichment (U-235) & & Normal & $3.90 \%$ & $4.00 \%$ & $3.95 \%$ \\
\hline 6 & $\begin{array}{l}\text { Enrichment (fissile } \\
\mathrm{Pu} \text { ) }\end{array}$ & & Normal & $59 \%$ & $60.2 \%$ & $59.6 \%$ \\
\hline 7 & Fuel temperature & $\mathrm{K}$ & Normal & 850 & 950 & 900 \\
\hline 8 & $\begin{array}{c}\text { Moderator } \\
\text { temperature }\end{array}$ & $\mathrm{K}$ & Normal & 557 & 661 & 559 \\
\hline 9 & Moderator density & $\mathrm{g} / \mathrm{cm}^{3}$ & Normal & -0.004 & +0.004 & $\mathrm{X}$ \\
\hline
\end{tabular}

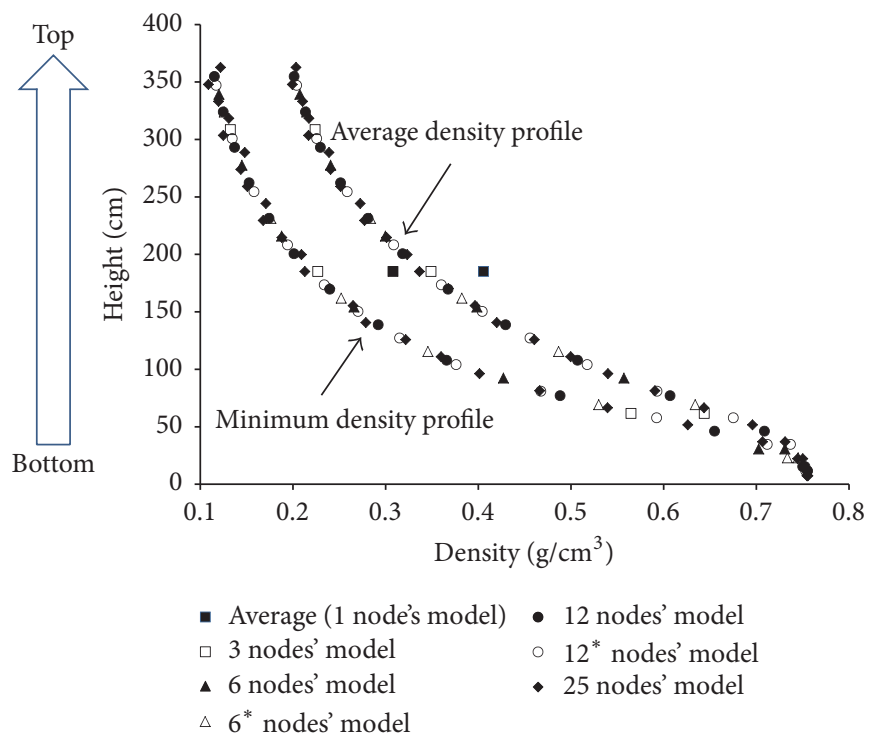

FIGURE 4: Distribution of moderator density values in simulations.

probability density function of moderator density is inversely proportional to the probability density function of moderator temperature.

The sensitivity and uncertainty analysis was performed for evaluation of void reactivity feedback and neutron multiplication factor for the cases with minimal and average moderator density profiles.

\section{Results}

3.1. Dependence of Void Reactivity Feedback on Fine Water Density Distribution. The moderator densities profiles used in this study consist of density values in 25 nodes (Figure 2). However, the description of density profiles across fuel assembly by dividing it to $1,3,6$, and 12 nodes per height and assigning appropriate values of moderator density for each separate node was considered. The additional models $\left(6^{*}\right.$ and $\left.12^{*}\right)$ were introduced in the analysis for the purpose of evaluating more precisely the rapid void formation in the top part of assembly. Denser numerical grid exists in the top part in comparison with the bottom part in these models. The average moderator density (moderation amount) per height was unchanged for both moderator density profiles (Figure 4).

Based on the comparison of neutron multiplication factors (Figure 5) it is seen that the application of 1 and 3 moderator values over active fuel length is not sufficient to describe the uneven distributions of two-phase flow to correspond to the close real conditions. The 1-node model underestimates $(\sim 1.4 \%)$ and 3 -node model overestimates $(\sim$ $0.1-0.3 \%)$ neutron multiplication factor in comparison with the results of reference density profiles (25-node model). 


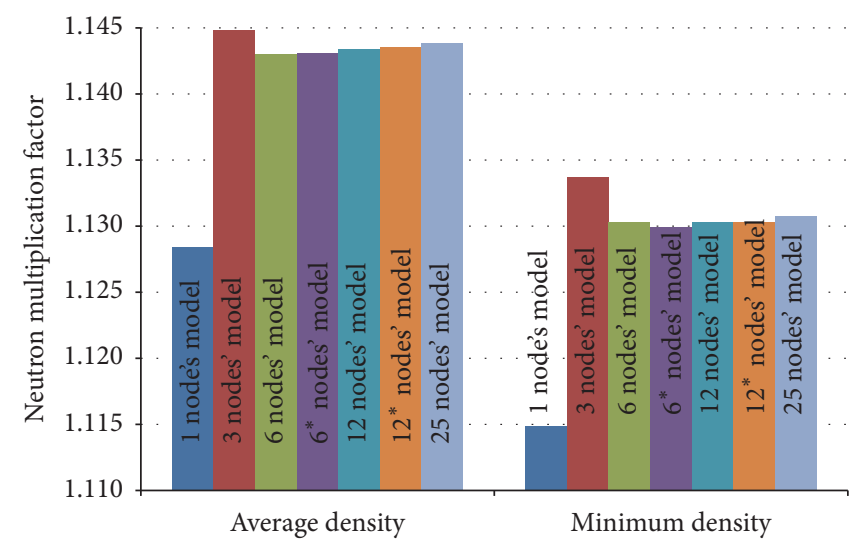

FIGURE 5: Neutrons multiplication factors calculated for separate density profiles.

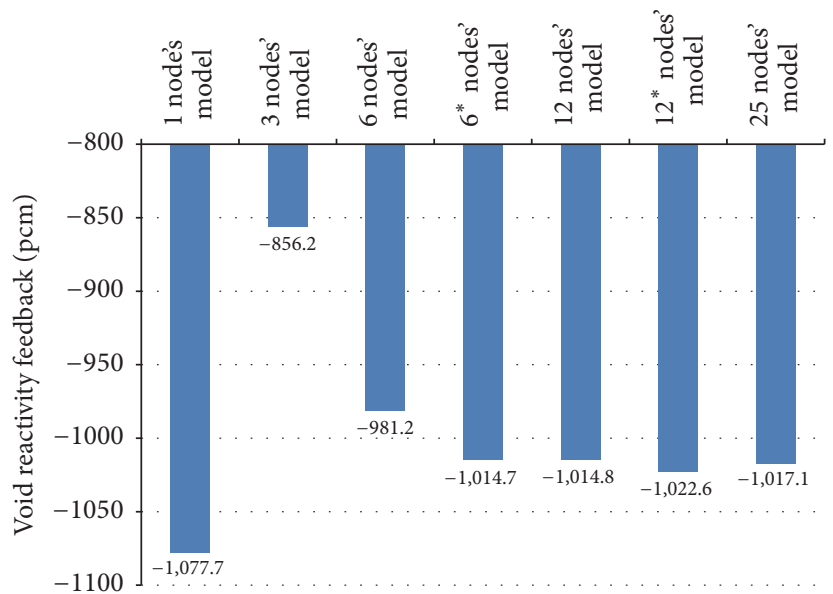

FIGURE 6: Void reactivity feedback calculated using different nodalisation schemes.

Meanwhile the predictions of other used density profiles correlate at high level: the discrepancy less than $0.1 \%$. Thus neutron moderation conditions across fuel assembly height can be described without significant deviation from close real conditions by using at least 6 moderator density values per FA height.

Void reactivity feedback which estimates reactivity difference between two states using minimal and average moderator density profiles is depicted in Figure 6 . It is seen that 1 -node model results in the overestimation by $6 \%$, while 3 node model underestimates $(\sim 16 \%)$ the void reactivity effect. Meantime the 3-node model can be considered as the most conservative approach. It can be considered as sufficient to simulate the void reactivity feedback applying 6-node model, as shown while analyzing the neutron multiplication factor. Nevertheless, denser $6^{*}$ nodes' model shows better congruence with reference case (deviation $2.4 \mathrm{pcm}$ only), and it was chosen for performing the detailed sensitivity and uncertainty analysis.

3.2. Sensitivity and Uncertainty Analysis. The application of the presented uncertainty analysis methodology for the investigation of void reactivity feedback provides quantitative uncertainty estimations in the form of two-side tolerance intervals. The empirical distribution functions for neutron multiplications factors and void reactivity feedback with twosided tolerance limit of $95 \%$ and for $95 \%$ confidence level are depicted in Figure 7.

The uncertainty ranges of neutron multiplication factors are $(1.116,1.132)$ and $(1.1305,1.146)$ for cases with average and minimal density profiles, accordingly. Empirical distribution functions for both profiles are of similar shape; thus it can be stated that the same sets of input data variables cause similar impact on criticality. It is seen that more than $80 \%$ of all predictions have less values than the reference case.

Values of neutron multiplication factors in cases of average and minimal density profiles overlap in the interval $(1.1306,1.1333)$. Nevertheless, the void reactivity feedback is negative all the time (Figure 7 ). The void reactivity feedback predictions deviate from reference value at maximum by $7 \%$. In comparison, the 1-node and 3-node models cause deviations by, accordingly, $-6 \%$ and $16 \%$ (Figure 6 ). Thus the selection of nodalisation scheme can cause even greater deviation in void reactivity feedback values than the uncertainties in input data variables. 


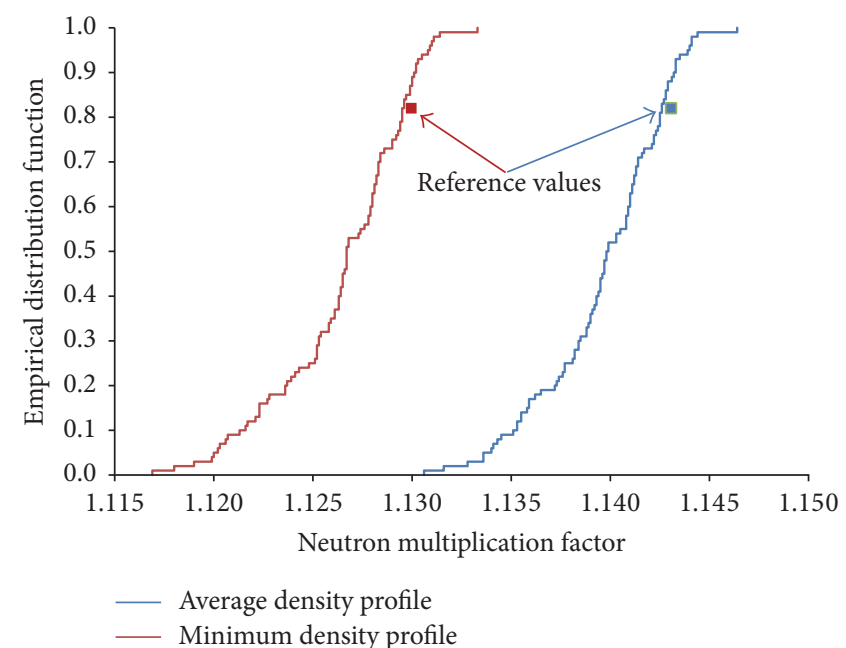

(a)

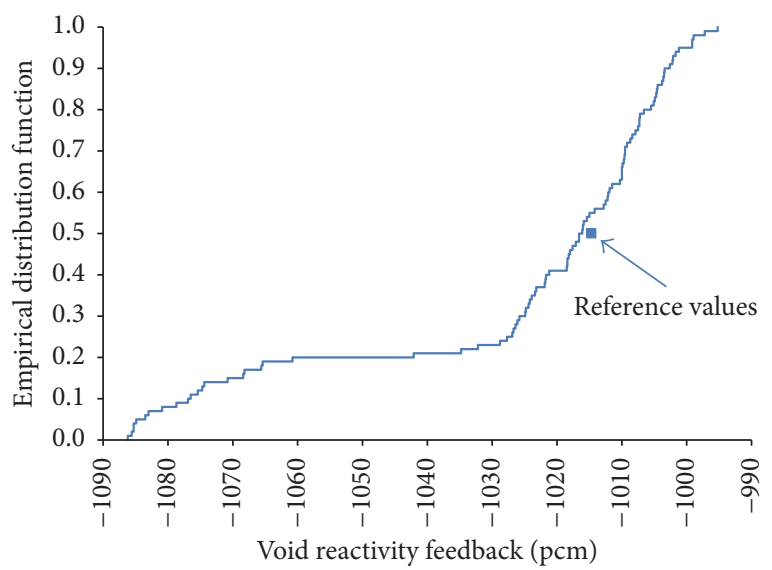

(b)

FIGURE 7: Scale uncertainty analysis results.

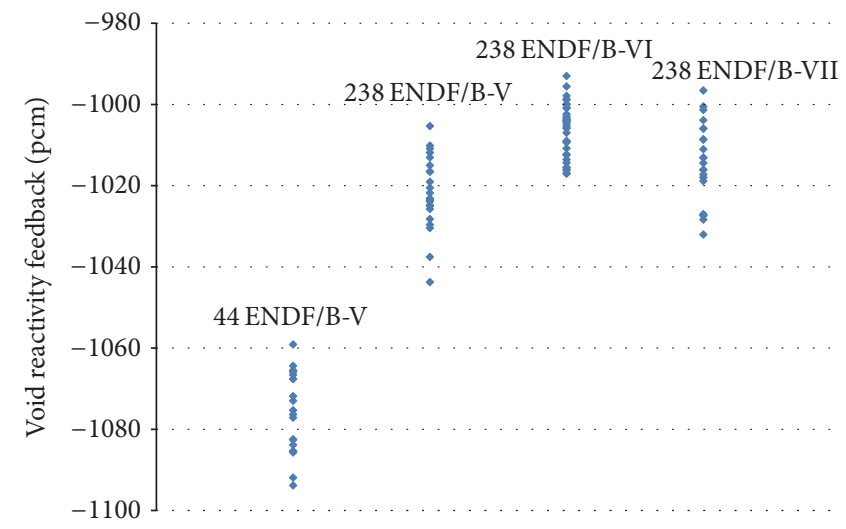

FIGURE 8: The correlation between the cross section library and void reactivity feedback.

The whole interval of void reactivity feedback predictions can be divided into 2 ranges: $(-1090,-1060) \mathrm{pcm}$, which include $20 \%$ of total predictions, and $(-1040,-990) \mathrm{pcm}$, which include $80 \%$, with no predictions between these intervals. It can be caused only by input data variable where uncertainty distribution is discrete, that is, the selection of cross section library. Figure 8 shows the influence of chosen cross section library. It is clearly seen that the predictions obtained using 44 groups ENDF/B-V library are different from all the others. Thus the selection of this data library for cross section processing causes higher negative values of void reactivity feedback.

It is possible to identify those input data variables with a larger influence in the variation of output variables while accounting for the variability of other inputs through their uncertainties. This can be done through the calculation of statistical sensitivity measures. Such analysis was accomplished in order to determine which parameters have the greatest influence on the uncertainty of neutron multiplication factor and void reactivity feedback. The SRCC used in this analysis isolates the influence of a given parameter with respect to the statistical influence of all the others.

SRCCs for multiplication factors and void reactivity feedback are depicted in Figure 9. The coefficient of multiple determinations $R^{2}$ is a global parameter that quantifies the statistical significance of the correlation coefficients computed for each output variable. The achieved high $R^{2}$ for neutron multiplication factors (0.7) and for void reactivity feedback (0.9) shows that there is strong linear correlation between input data variables and estimated results. Only input data variables, SRCC of which is $>0.2$, are considered as having a recognizable influence.

Similar distributions of SRCCs are estimated for both used moderator density profiles. The uncertainty of neutron multiplication factor strongly depends upon cross section library (parameter number 1), fuel pellet diameter (number 2 ), fuel pellet density (number 4), and cladding diameter (number 3). Meanwhile the uncertainties of fissile material 


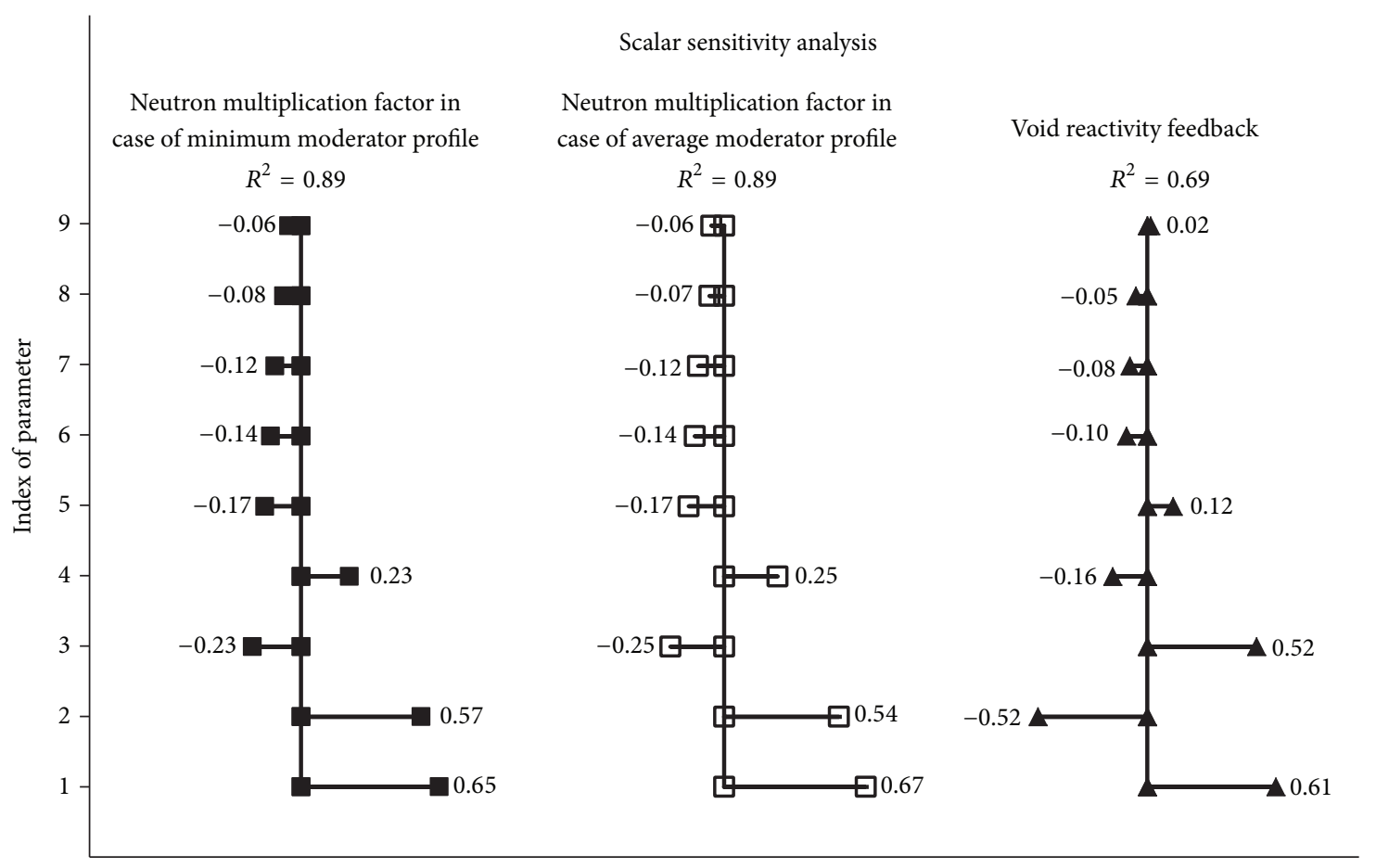

Spearman's partial rank correlation coefficients

FIGURE 9: Results of scalar sensitivity analysis.

enrichments, fuel temperature, moderator temperature, and density have no recognizable influence. Neutron multiplication factor has the positive dependence on the selection of cross section library, fuel pellet diameter, and fuel density. The use of 238-group libraries in place of 44-group library results in increase of neutron multiplication factor for both cases of density profiles. Thus it can be stated that the structure of 44 energy groups makes the neutron spectrum harder, in comparison with 238 energy group libraries. The increases of fuel pellet diameter and fuel density result in the change of fuel amount, which, respectively, increases the multiplication factor. It must be noted that the uncertainties of fuel pellet diameter and fuel density call in more greater changes of fissile material quantity than the uncertainties of fissile material enrichments, since SRCCs for the enrichment of U-235 and the enrichment of $\mathrm{Pu}$ fissile isotopes are comparatively smaller $(-0.17$ and -0.14 , accordingly). The change of cladding diameter has negative influence on neutron multiplication factor since with increase of cladding thickness (outer cladding diameter) the amount of moderator is decreasing. In addition, less neutrons participate in chain reactions due to the increase of nonfissile neutrons absorption in cladding.

Talking about void reactivity feedback, it significantly depends on the selection of cross section libraries (parameter number 1), fuel pellet (number 2), and cladding (number 3 ) diameters (Figure 9).

All BWR reactors must be undermoderated because such conditions guarantee a negative void reactivity feedback. Since the void reactivity feedback is negative in interval of all predictions (Figure 7) it is evident that the analyzed system is undermoderated. The decrease of moderator/fuel ratio (hardening neutron spectrum) results in the decrease of void reactivity feedback in comparison to reference moderation conditions as the system undergoes even less moderated conditions (see Figure 3). And, conversely, the increase of moderator/fuel ratio (softening of neutron spectrum) results in increase of void reactivity feedback as system is approaching overmoderated conditions. Thus scalar sensitivity analysis results of void reactivity feedback can be explained through the influence of input variables uncertainties on moderations conditions.

If fuel pellet diameter increases the moderator/fuel ratio decreases. Thus it significantly influences larger negative values of void reactivity feedback. Positive SRCC for parameter number 1 shows that the use of 238-group library in place of 44-group library results in softer neutron spectrum since the absolute values of void reactivity feedback decrease. It can be stated that the 44-group data library can be an insufficient representation of its parent 238-group library since a more detailed group structure or a more appropriate collapsing spectrum are needed for accurate estimation of the void effect. The increase of cladding diameter makes the neutron spectrum harder as well since the moderation amount decreases. As a consequence, the void effect decreases.

\section{Conclusions and Discussions}

The uncertainty and sensitivity analysis considering the estimation of void reactivity feedback is accomplished. Void 
reactivity feedback was simulated between two selected cases based on fine moderator axial density profiles. The minimum and average density profiles, which represent the conservative and average conditions during normal operation, were selected for the analysis. The study of different nodalisation schemes for moderation conditions description across fuel assembly height revealed that moderator density profile with $6^{*}$ nodes can be considered sufficient to simulate void reactivity feedback since its prediction is close enough (deviation only $2.4 \mathrm{pcm}$ ) to the result of reference profile, consisting of 25 values across fuel height $(3.7 \mathrm{~m})$.

The uncertainty analysis showed that the void reactivity feedback is negative in the interval of all predictions. Despite the overlapping of neutron multiplication factor's uncertainty ranges, the values of neutron multiplication factor in case of average density profile are larger than the values in case of minimum density profile in all the same sets of variable input data samples. In addition, it was demonstrated that the selection of nodalisation scheme can cause even greater deviation in void reactivity feedback than the uncertainties in input data variables.

The uncertainty of neutron multiplication factor strongly depends on the selection of cross section library, fuel pellet diameter and density, and cladding diameter. Meanwhile the uncertainty of void reactivity feedback is influenced by uncertainties of the same input parameters with exception of the fuel density.

It is clearly seen that the predictions with selected 44group ENDF/B-V library are distinguished from all other predictions. The 44-group data library is an insufficient representation of its parent 238-group library for MOX fuel, as it gives overpredicted results, in comparison to parent library.

\section{Conflicts of Interest}

The authors declare that there are no conflicts of interest regarding the publication of this article.

\section{References}

[1] IAEA-TECDOC-1654, Advanced Fuel Pellet Materials and Fuel Rod Design for Water Cooled Reactors, IAEA, Vienna, 2010.

[2] F. Jatuff, F. Giust, J. Krouthén, S. Helmersson, and R. Chawla, "Effects of void uncertainties on the void reactivity coefficient and pin power distributions for a $10 \times 10 \mathrm{BWR}$ assembly," Annals of Nuclear Energy, vol. 33, no. 2, pp. 119-125, 2006.

[3] G. Alonso, S. Bilbao, and E. Del Valle, "Impact of the moderation ratio over the performance of different BWR fuel assemblies," Annals of Nuclear Energy, vol. 85, pp. 670-678, 2015.

[4] A. Slavickas, R. Pabarčius, A. Tonkunas, and E. Ušpuras, "Analysis of Void Reactivity Coefficient for 3D BWR Assembly Model," Science and Technology of Nuclear Installations, vol. 2016, Article ID 3427549, 2016.

[5] A. Inoue, T. Kurosu, T. Aoki, M. Yagi, T. Mitsutake, and S.-I. Morooka, "Void Fraction Distribution in BWR Fuel Assembly and Evaluation of Subchannel Code," Journal of Nuclear Science and Technology, vol. 32, no. 7, pp. 629-640, 1995.
[6] W. J. Marshall, B. J. Ade, S. M. Bowman, and J. S. MartinezGonzalez, "Axial moderator density distributions, control blade usage, and axial burnup distributions for extended bwr burnup credit," Tech. Rep. (ORNL/TM-2015/544), Oak Ridge National Laboratory, Oak Ridge, Tenn, USA, 2016.

[7] M. Pecchia, G. Kotev, C. Parisi, and F. D'Auria, "MOx benchmark calculations by deterministic and Monte Carlo codes," Nuclear Engineering and Design, vol. 246, pp. 63-68, 2012.

[8] J. L. Francois and C. Martin del Campo, "Lattice physics codes comparisons for the NEA BWR MOX benchmark," in Proceedings of the International Topical Meeting on Advances in Reactor Physics and Mathematics and Computation into the Next Millennium, Pittsburgh, PA, USA.

[9] M. Kloos, SUSA Version 4.0, Software for Uncertainty and Sensitivity Analyses, User's Guide and Tutorial, GRS-P-5, Rev. 2, Gesellschaft für Anlagen- und Reaktorsicherheit (GRS) mbH, Garching, Germany, 2016, (saved in file "GRS - P - 5 Rev2.pdf" of the installation package).

[10] S. S. Wilks, "Statistical prediction with special reference to the problem of tolerance limits," Annals of Mathematical Statistics, vol. 13, pp. 400-409, 1942.

[11] OECD NEA, "Evaluation Guide for the Evaluated Spent Nuclear Fuel Assay Database (SFCOMPO)," Tech. Rep. NEA/NSC/ R(2015)8, 2016. 


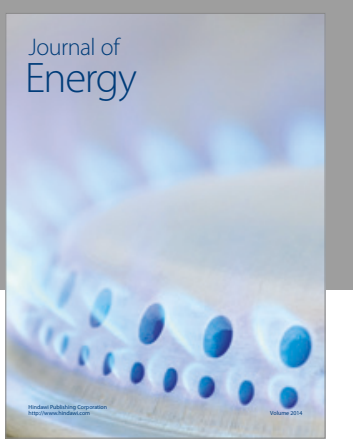

Journal of

Industrial Engineering
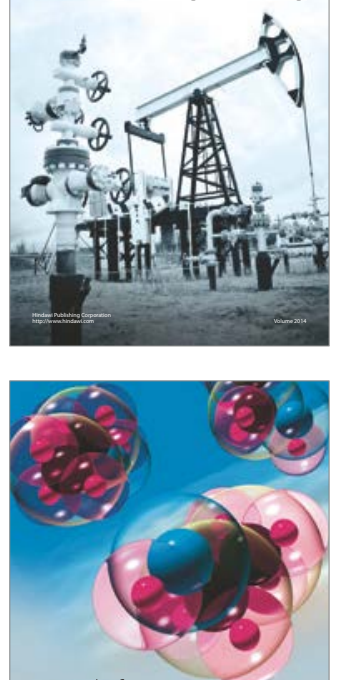

Fuels
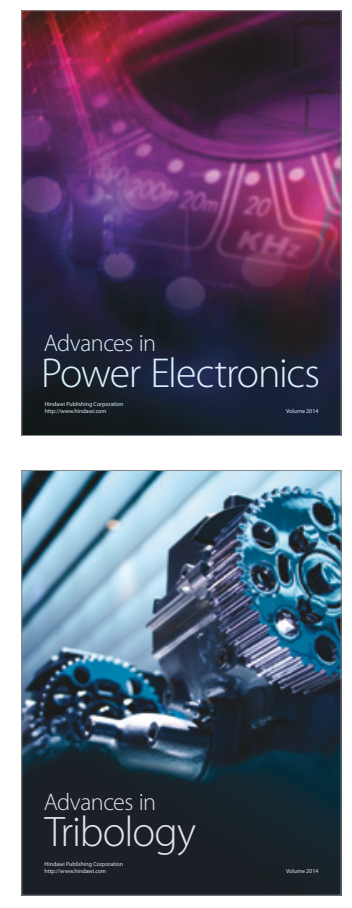
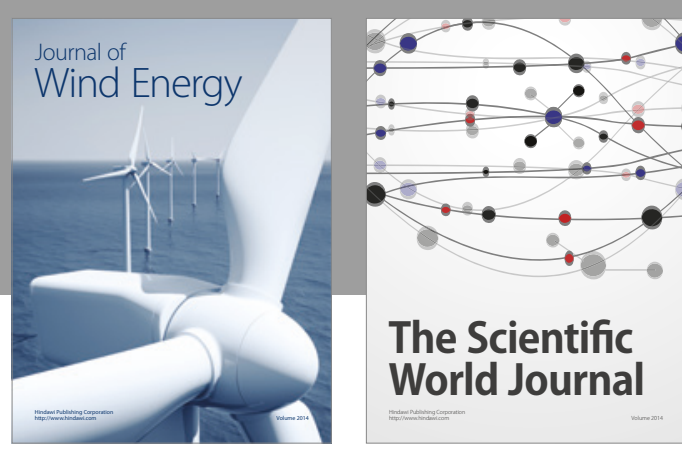

The Scientific World Journal
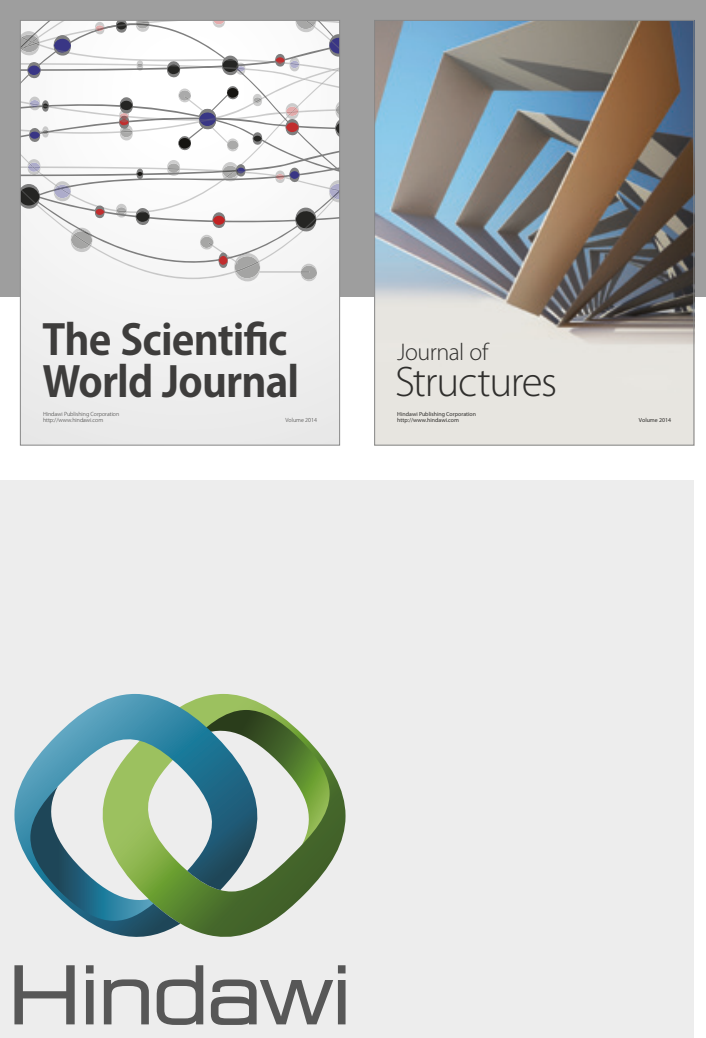

Submit your manuscripts at

https://www.hindawi.com
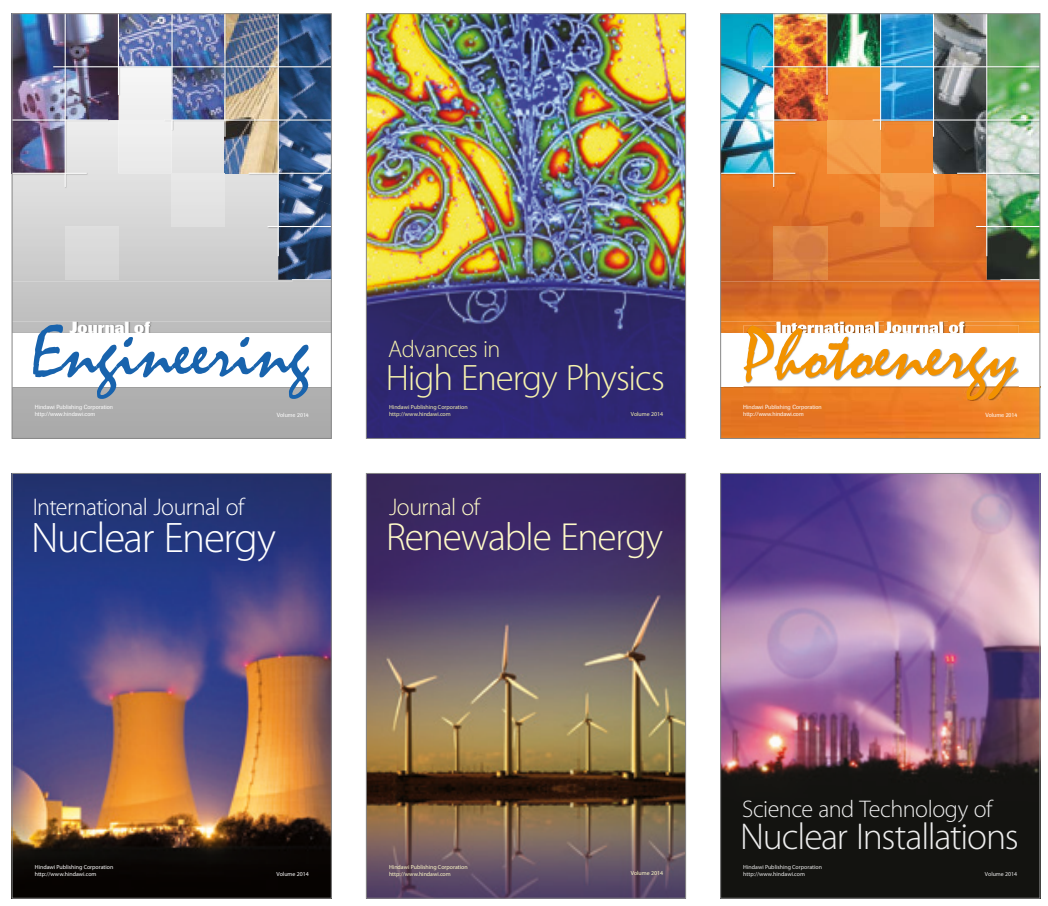

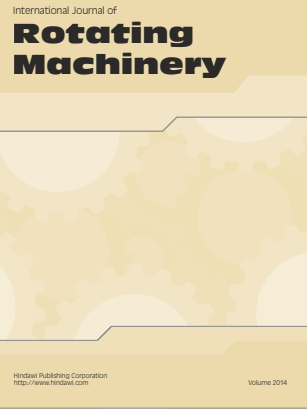

Journal of

Petroleum Engineering

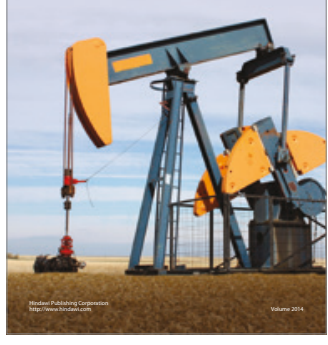

Journal of
Solar Energy
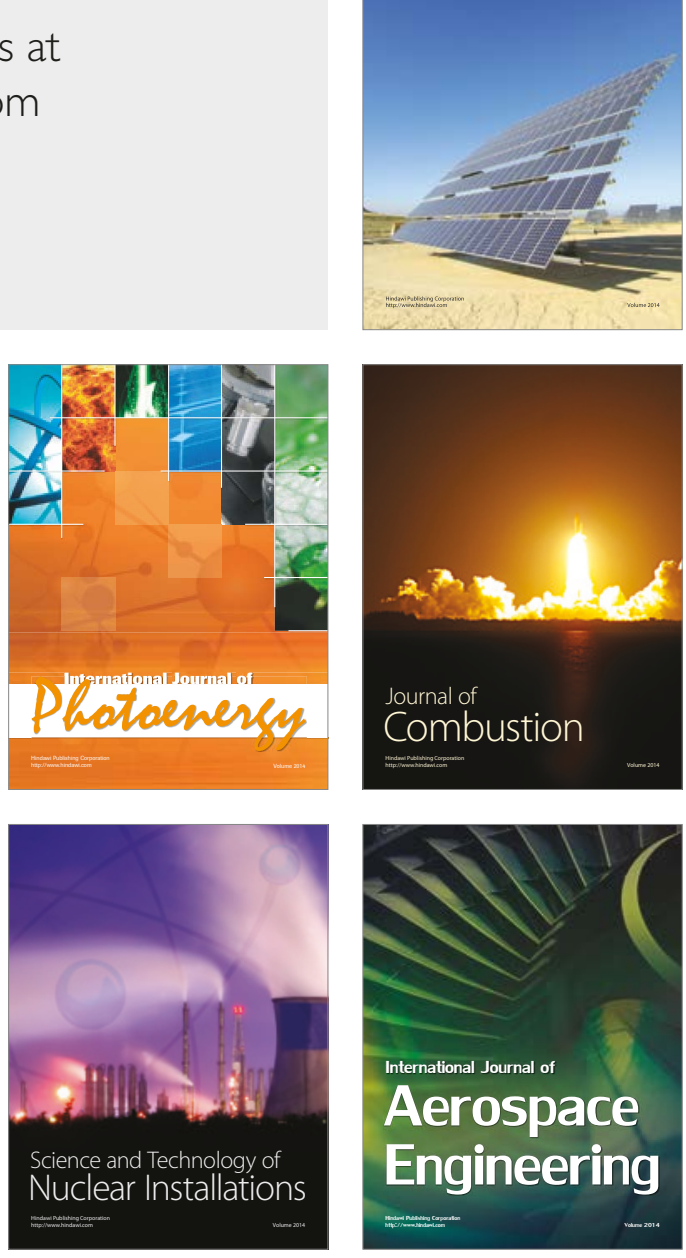